\title{
Culturing our sense of smell
}

\author{
JENNIFER ROBERTSON
}

\section{Abstract}

The functioning and connectivity of the olfactory system, which produces our sense of smell, has recently been attracting increased attention from neuroscientists, particularly since the award of the 2004 Nobel Prize to Buck and Axel for their research in this field. To study the olfactory system further, it would be advantageous to develop methods for keeping isolated brain tissue viable for long periods of time. This study examines the morphology, electrophysiology and survival of cells in brain slices that have been kept in culture, in comparison to acute slices. We prepared parasagittal slices that contained the olfactory bulb (OB), the piriform cortex (PC) and the lateral olfactory tract (LOT). Such slices would be particularly useful in studies that depend upon intact connectivity between the OB and the PC. In this study, we identified differences in the synaptic and intrinsic properties between pyramidal neurons in the acute and cultured slices. Also, the GABAergic interneurons appear not to have been affected. Therefore, we concluded that these slices would be suitable for use in studying some aspects of the olfactory system but only once the differences identified have been investigated further.

\section{Introduction}

Despite decades of research, much is still unknown about how the olfactory system functions, particularly how information is processed in the PC (also known as the primary olfactory cortex) (Kandel 2000). Recently, scientists such as Buck and Axel (1991) have demonstrated that any given odour receptor can recognise many different ligands and any odour ligand can bind to many different receptors, resulting in almost unlimited specificity (Firestein 2005, Dennis 2004). The question then remains, how does the brain process all of these signals to recognise a single smell? Researchers are now focusing on this question. It has been demonstrated that the PC is the first site of cortical 
processing and thus a major area of interest (Poo 2009, Sheperd 2006). It is believed that understanding how information is processed in this relatively simple part of the cortex, will enable understanding of more complex, cortical regions (Shepherd et al. 2006).

A common technique for studying the olfactory system is to prepare acute slices and measure the properties and connectivity of the neurons, within the slice, using patch clamping. However, this technique is limited by the approximately 5 hour lifespan of of the neurons once they have been removed from the brain. Therefore, there have been few studies on the connectivity changes or the molecular biology in this region, as such studies require more time. Thus, there is the requirement of a technique that can maintain the slices for longer. This study assesses the use of cultured slices to fulfil this requirement.

The slices of particular interest to this study are parasagittal slices that encompass the $\mathrm{OB}$, the $\mathrm{PC}$ and the interconnecting LOT (Figure 1). These slices are of particular interest because if suitable, they will allow researchers to study the connections between the $\mathrm{OB}$ and the PC. Understanding how the signal is changed in between these locations will provide insight into how olfactory information is processed.
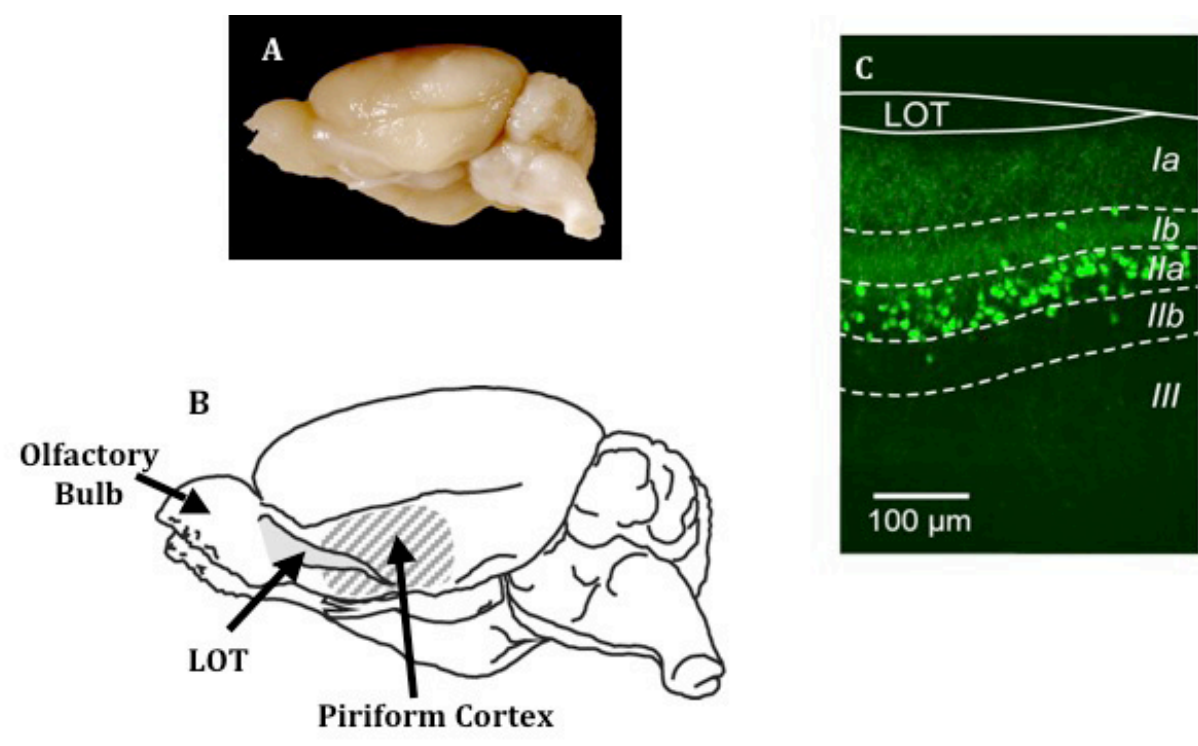

Figure 1: $A$ : A mouse brain $B$ : A schematic of a rodent brain demonstrating the OB, LOT and PC, the line represents the slices used for this study $C$ : The layers of the PC.

Figures adapted from images by Bekkers 
The method of culturing slices of mice brains has proven successful for hippocampal slices with minimal differences to the acute slices (Stoppini et al. 1991). Here we apply this method for the first time to parasagittal PC slices. We tested these slices by examining the morphology of individual stained neurons, testing them electrophysiologically and observing any changes in the fluorescence and soma size of GABAergic interneurons in the culture. It was hypothesised that the cultured slices would give similar results to the acute slices, but in our study some differences were identified.

\section{Materials and Methods}

\section{Slice Preparation}

The brains of P7 GAD67-GFP transgenic mice (cultured slices) and a P25 GAD67GFP transgenic mouse (acute slice) were aseptically removed in accordance with the Animal Experimentation and Ethics Committee of the Australian National University. GAD67-GFP transgenic mice are mice that express a green

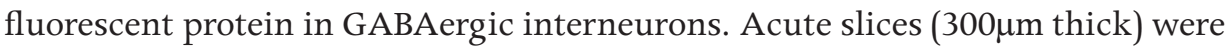
prepared using standard methods (Suzuki and Bekkers, 2010). For cultured slices, parasagittal slices $(200 \mu \mathrm{m})$ were taken using a razor blade in a sterilised, oxygenated ice-cold solution of (in $\mathrm{mM}$ ) $1 \mathrm{CaCl} 2,5 \mathrm{MgCl} 2,10$ glucose, $4 \mathrm{KCl}$, $26 \mathrm{NaHCO} 3,248$ Sucrose and phenol red (acidity indicator). The slices from the P7 mice were then transferred to the culture dish and those from the P25 mouse were used immediately or fixed. This allowed for the slices to be of a similar age when analysed.

\section{Slice Culture}

The slices were placed on semiporous membranes in a 6 well culture dish. Each well contained, $0.75 \mathrm{~L}$ of culture medium (containing 0.0084g/mL MEM in Hepes base, $20 \%$ horse serum, $1 \mathrm{mM} \mathrm{L-glutamine,} 1 \mathrm{mM} \mathrm{CaCl}{ }_{2^{\prime}} 2 \mathrm{mM} \mathrm{MgSO}_{4^{\prime}}$ $12.9 \mathrm{mM}$ D-Glucose, 5.2 $\mathrm{mM} \mathrm{NaHCO}{ }_{3}, 30 \mathrm{mM}$ Hepes, $0.075 \%$ Ascorbic acid, and $1 \mu \mathrm{g} / \mathrm{mL}$ Insulin). The culture was kept at $35^{\circ} \mathrm{C}$ in $5 \% \mathrm{CO} 2$, for 5-7 days and fed every 2-3 days.

\section{Electrophysiology}

The slices were studied using patch clamp techniques. They were maintained in a bath solution of artificial Cerebral Spinal Fluid (CSF), which was kept at around $35^{\circ} \mathrm{C}$ and bubbled with carbogen (95\% oxygen, $5 \%$ carbon dioxide). The 
activity of individual neurons in the slices were recorded using electrodes filled with internal solution containing (in mM) $135 \mathrm{~K}$-methylsulfate, $7 \mathrm{KCl}, 0.1$ EGTA, $2 \mathrm{~N}_{\mathrm{a}} 2 \mathrm{ATP}, 2 \mathrm{MgCl}_{2}, 0.3 \mathrm{Na}_{2} \mathrm{GTP}, 10$ Hepes and $0.2 \%$ biocytin. Cells were chosen from Layer II (Figure 1C) that appeared to be pyramidal neurons (from somatic morphology). Whole-cell recordings were taken of neurons using a MultiClamp 700 amplifier and Axograph. The passive and firing properties of individual neurons were determined by applying a series of positive and negative current steps using current clamp. Next we inserted a stimulating electrode into the LOT and recordings of EPSPs (excitatory post-synaptic potentials) were taken. This was repeated with the stimulating electrode in Layer II. The pipette offset was monitored and adjusted, as were the resting membrane potential and the bridge balance. A holding current was applied to some cells to maintain the resting membrane potential at approximately $-65 \mathrm{mV}$.

\section{Histology}

The recording electrode contained $0.2 \%$ biocytin, which diffused throughout the neuron during the recordings. The slices were then fixed using $4 \%$ paraformaldehyde for 1 hour and washed with Phosphate Buffered Saline (PBS). The slices were then processed using a standard biotin-avidin method (Horikawa 1988). The slices were washed with PBS then distilled H2O and mounted onto microscope slides. The stained cells were observed under an electronic microscope using a $63 \mathrm{x}$ oil immersion objective and traced using the Neurolucida cell reconstruction system (MBF Bioscience).

\section{Fluorescence Studies}

The remaining cultured slices were removed from culture and fixed using 4\% paraformaldehyde for 1 hour and washed with PBS. They were then mounted and $5 \mu \mathrm{m}$ image stacks of the GFP fluorescence through each slice were taken using a confocal microscope. Each soma was traced in the optical section where the fluorescence of the soma was the brightest. The fluorescence and soma size was recorded for each neuron.

\section{General Analysis}

The electrophysiological data was analysed using Axograph and Excel. Due to the small number of cells, a large number of recordings were taken from each cell and the means of each analysis were compared graphically. Recordings were excluded from the mean if they contained major irregularities due to the spontaneous or epileptic activity of the neuron. Due to the small number of cells, further statistical analysis was not performed. 


\section{Analysis of Intrinsic Properties}

To determine if the cells maintained the same basic electrophysiological properties after culture, the standard intrinsic properties were measured. These were: the membrane time constant (the product of the input resistance and capacitance of the membrane), the resting membrane potential (difference in electrical charge across the membrane when at rest), the input resistance (relationship between current injected into the cell and the resulting voltage), the voltage threshold (the minimum voltage required for the action potential to fire) and the action potential half-width (the width of the action potentials at half their height) (Kandel, 2000).

The membrane time constant was calculated from the exponential of best fit to the voltage decrease, which occurred after a small current step. The resting membrane potential was measured as the voltage of the neuron before the current step was applied. The input resistance was calculated from the graph of voltage and current using Ohm's Law (V=IR). The voltage threshold was measured as the voltage at which the action potential fired. The spike half-width was determined by measuring the width of the action potential at half its height.

\section{Analysis of Synaptic Properties of Pyramidal cells}

To determine if the cells responded to stimulation in the same way, we tested the synaptic properties of pyramidal cells. A stimulating electrode was inserted first into the LOT and then into Layer II of each of the slices. In each case the stimulus was a series of five pulses of $20 \mathrm{~Hz}$. The response of the cells to each series of stimulations was recorded 51 times for each location (to reduce overall error) and a final measurement was recorded again at the LOT (1st position). The similarity between the initial and final recordings showed that the results were not merely due to the reinsertion of the stimulating electrode.

The amplitude of the EPSPs was normalised to the size of the initial peak in each recording and a baseline correction was made at the base of each peak.

From the EPSP recordings it was possible to determine the EPSP decay time by fitting an exponential to the final EPSP (the others were affected by summation) and the synaptic latency by measuring the time between the stimulus artefact and where the first EPSP began We compared the differences in synaptic latency and decay between the LOT and Layer II in the acute slice and the two cultured slices. 


\section{Analysis of Fluorescence Data}

Normalised frequency histograms were constructed for both soma size and fluorescence intensity using the data from our cultured slice recordings and previously published data from acute slice recordings (Suzuki and Bekkers, 2010). The mean and standard error of each were also calculated.

\section{Results}

In this study we recorded the morphology and the intrinsic membrane and synaptic properties of one pyramidal neuron from an acute slice and two from cultured slices. The fluorescence studies were conducted on two cultured slices and compared to data from acute slices from Suzuki and Bekkers, 2010.

The neurons in both the cultured and acute slices have a broadly pyramidal morphology.
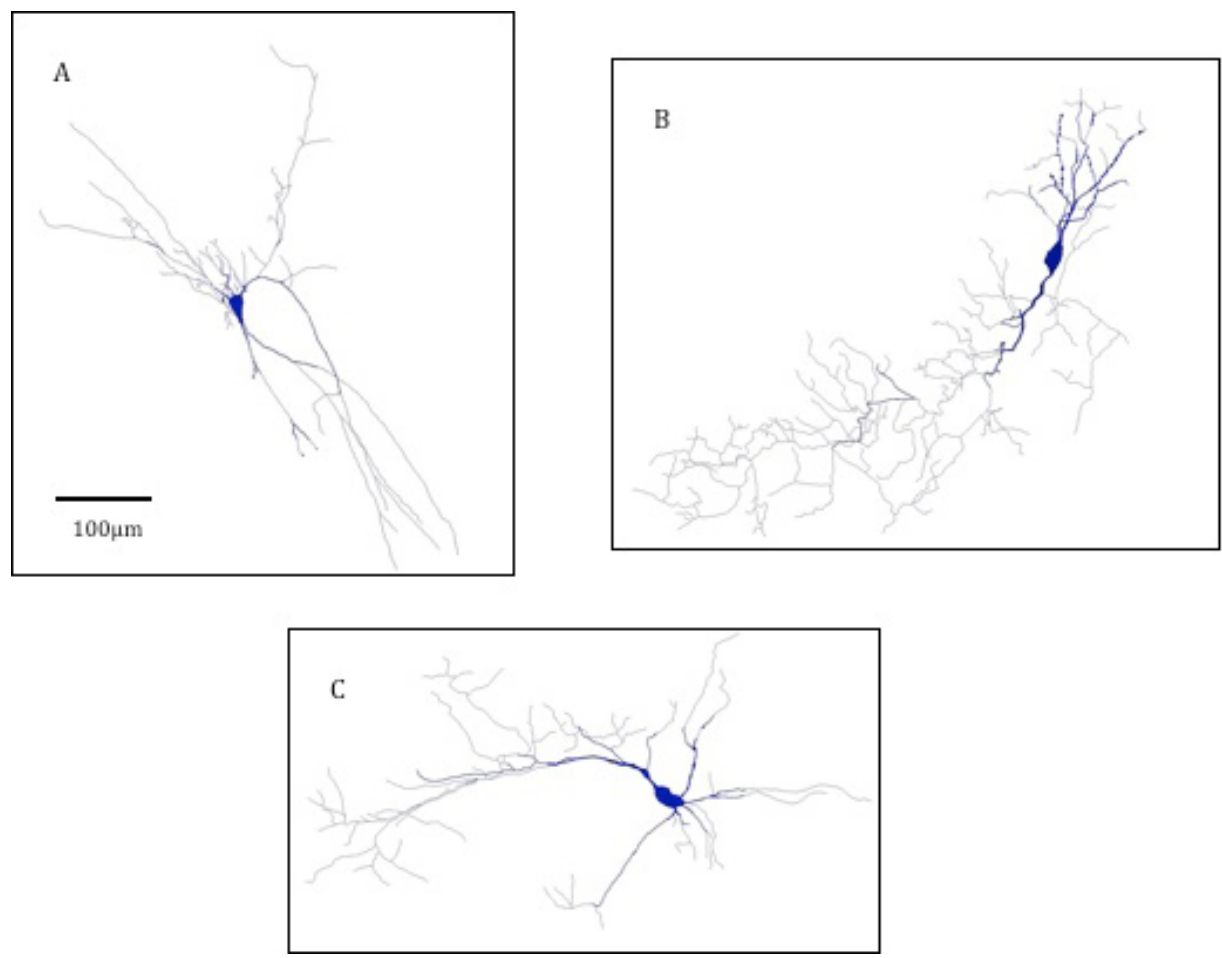

Figure 2: Tracings of the analysed neurons. A: The neuron from the acute slice. B: The neuron from cultured slice 1. C: The neuron from cultured slice 2. 
The tracings of the neurons were completed using the Neurolucida cell reconstruction system and allowed for a comparison of the morphology (Figure 2). All the neurons can be tentatively described as pyramidal neurons. There are, however, several irregularities. The variability in soma shape and size may have occurred from the insertion of the recording electrode. There are differences in the amount of branching, the length and the distribution of the dendrites. Due to the small number of cells traced and time constraints, these differences could not be quantified.
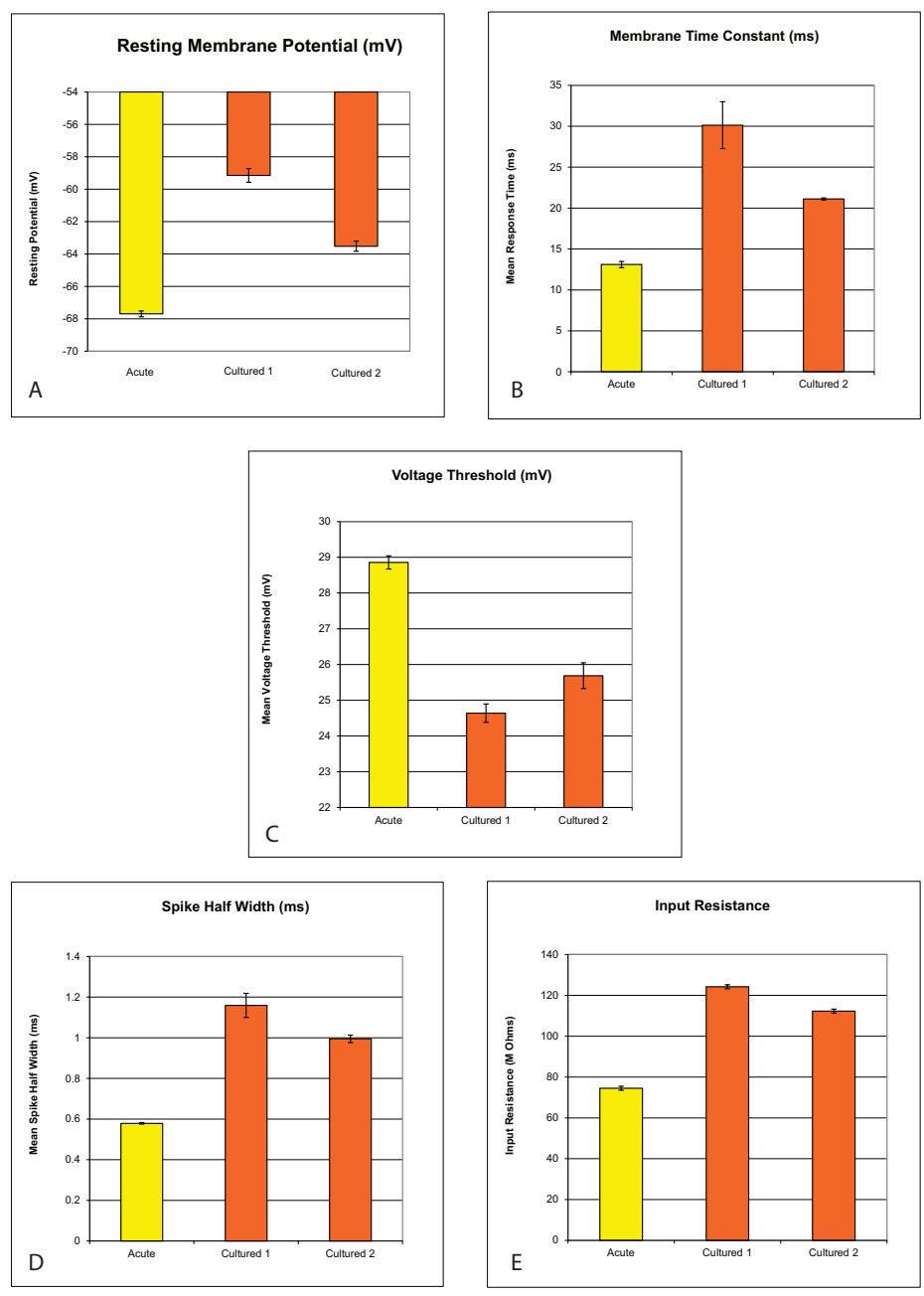

Figure 3: Comparisons of the mean intrinsic properties (error bars show standard deviation) of the neurons in the acute slice (yellow bars) and cultured slices (orange bars). A: resting membrane potential, B: membrane time constant, C: voltage threshold for action potential generation, D: spike half-width, and $\mathrm{E}$ : input resistance 
The neurons in the cultured slices exhibit different intrinsic properties to that of the acute slice.

After confirming that there was little change in the morphology, we focused on comparing the intrinsic membrane properties of resting membrane potential, membrane time constant, voltage threshold, spike half-width and input resistance (Figure 3).

Despite being unable to complete a further statistical analysis on the intrinsic properties, (sample sizes being too small), it can be observed from the graphs that there is a large difference in the membrane time constant, input resistance, resting membrane potential, voltage threshold and spike half-width in the cultured slices compared to the acute slice. There is also a difference between the cultured slices, which suggests more cells need to be analysed.

\section{The neurons in the cultured slices and acute slices exhibit some differences in synaptic properties.}

To determine if the connectivity of the neurons was maintained in culture, despite the intrinsic properties changing, a study of the synaptic properties was undertaken.

When the stimulating electrode is located in the LOT (Figure 4), there are significant differences in the EPSP responses from the acute and cultured slices. In the acute slice, the EPSPs facilitate (i.e. the size of the EPSPs increases over the first two or three stimuli). However, in the cultured slices, the EPSPs do not. This suggests a difference in the synaptic connectivity.

When the stimulating electrode is located in Layer II, the EPSP responses of the neurons are very similar in the acute and cultured slices (Figure 5); there is no facilitation. Thus, unlike the LOT input (Figure 4), there has not been a change in the synaptic properties, so it is likely that the connections are still the same. 


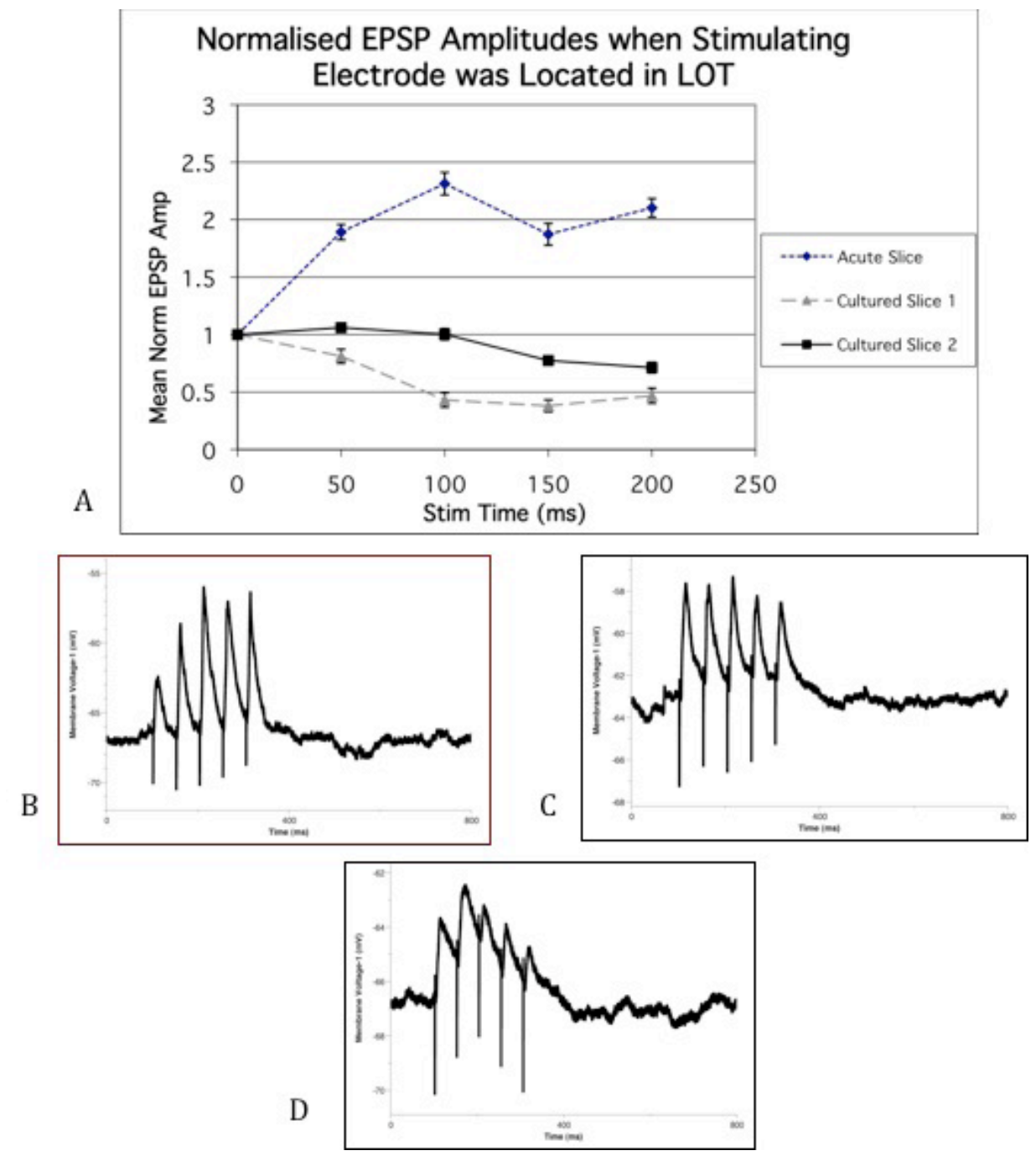

Figure 4: The EPSPs in the acute slice exhibit facilitation during LOT stimulation but those from the cultured slices do not. A: Average normalised EPSP amplitude comparison. B: Typical recording from the acute slice neuron. C: Typical recording from the cultured slice 1 neuron. D: Typical recording from the cultured slice 2 neuron. In panels B, C and $D, X$-axis shows time from $0-800$ milliseconds and the $Y$-axis shows the mebrane voltage in millivolts. 

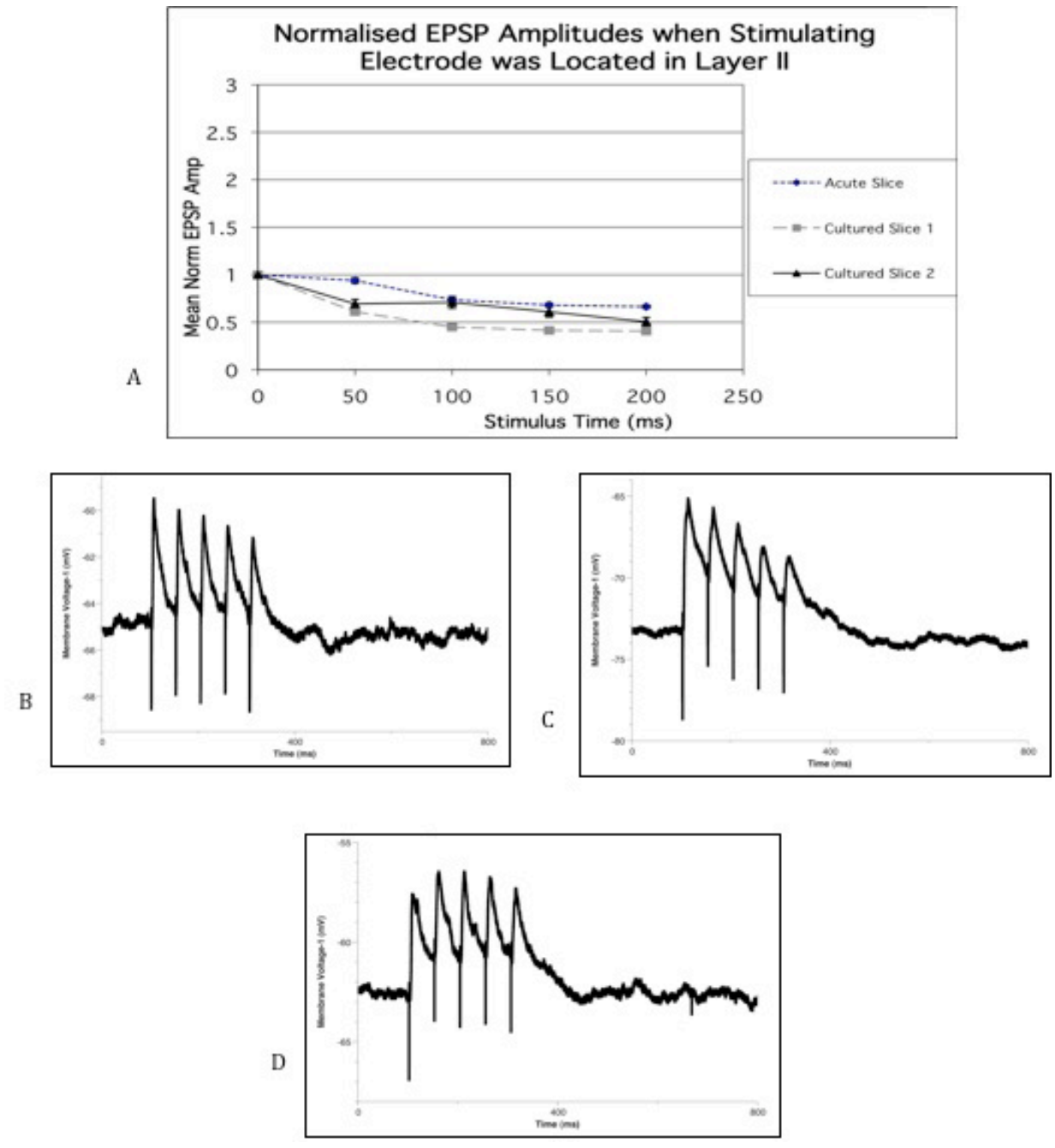

Figure 5: The EPSPs in the acute slice exhibit facilitation during Layer II stimulation but those from the cultured slices do not. A: Average normalised EPSP amplitude comparison. B: Typical recording from the acute slice neuron. $C$ : Typical recording from the cultured slice 1 neuron. D: Typical recording from the cultured slice 2 neuron. In panels B, C and $D, X$-axis shows time from $0-800$ milliseconds and the $\mathrm{Y}$-axis shows the mebrane voltage in millivolts.

Having identified a possible change in the synaptic properties we attempted to determine if the lack of facilitation in the cultured slices during LOT stimulation was due to a change in connectivity of the synapses in culture. We measured the synaptic latency and EPSP decay to do this. The latency comparison (Figure $6 \mathrm{~A})$, shows a large difference between the acute slice and cultured slice 1 , as well 
as the acute slice and cultured slice 2. The decay time comparison, (Figure 6B) shows differences between the acute slice and cultured slice 1 but not cultured slice 2 . This suggests that these differences may just be the result of intercellular variability.

\section{Synaptic Latency Comparison}

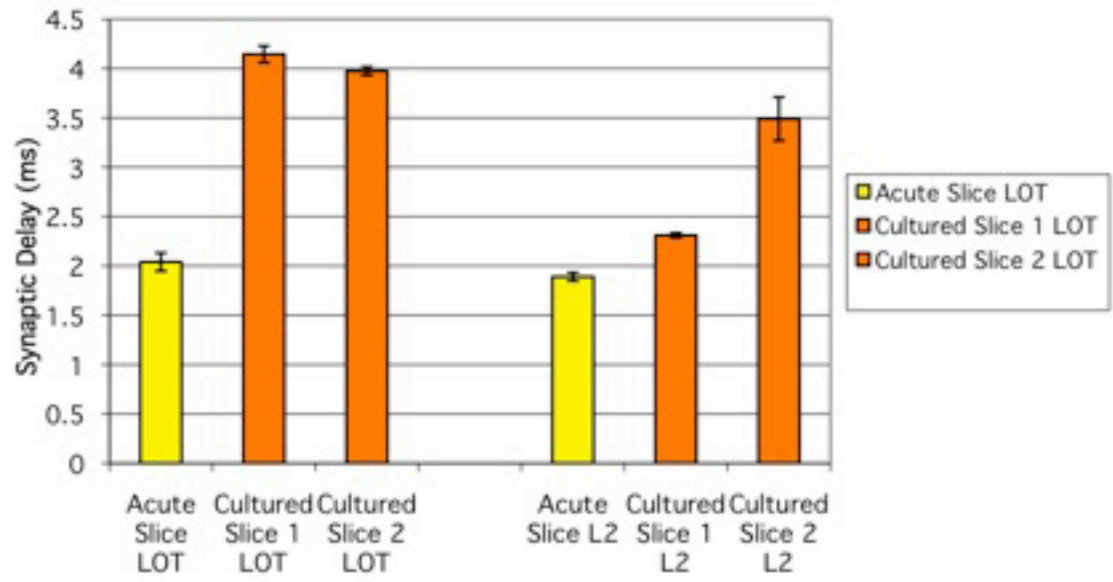

\section{Decay Time Comparison}

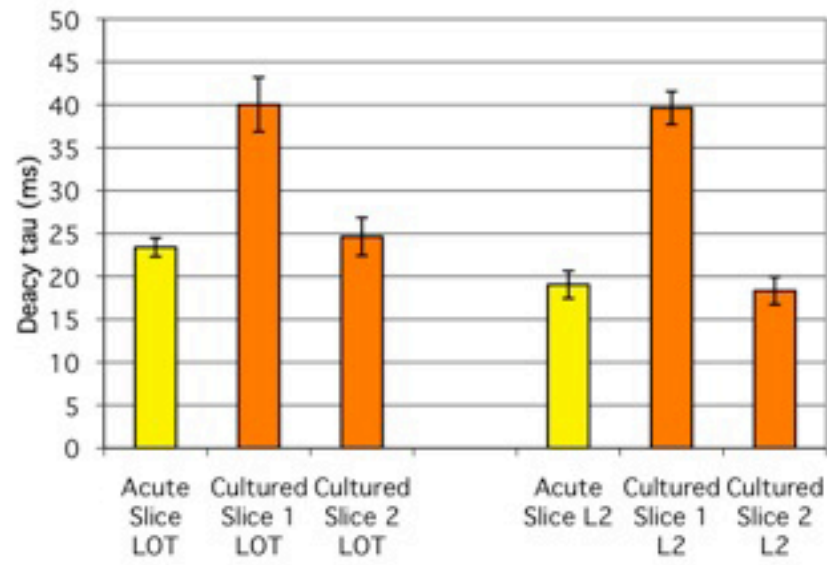

Figure 6: A. Comparison of the EPSP latency in the acute and cultured slices in the LOT and Layer II. B: Comparison of the decay in the acute and cultured slices in the LOT and Layer II. 
The subtypes of GABAergic interneurons remained unchanged in culture.

Since there are similarities and differences in the morphology, intrinsic properties and synaptic properties of the pyramidal neurons, have there been any changes in GABAergic interneurons? We attempted to answer this question through a comparative study of both the fluorescence intensity and soma size of GABAergic interneurons, between the cultured and acute slices. Figure 7 demonstrates the fluorescence of the slice and the slice under white light.
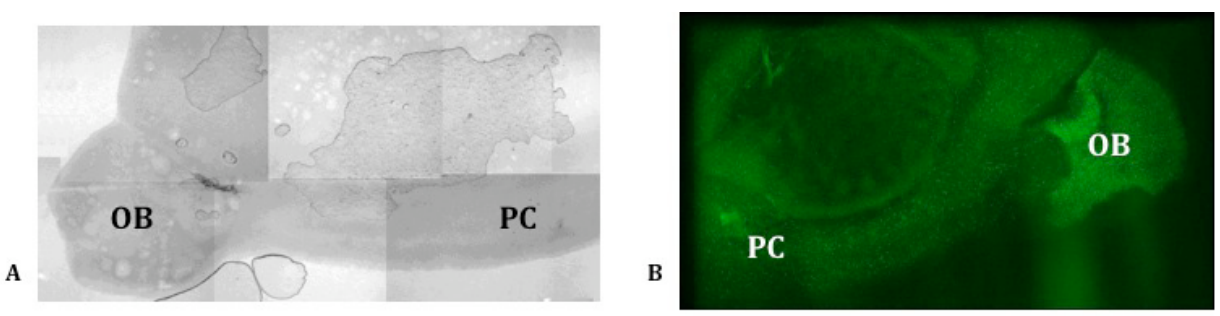

Figure 7: A: A reconstructed image of a cultured slice under white light and $B$ : An image of a cultured slice demonstrating the green fluorescence of the GABAergic somata.

The fluorescence intensity histograms for the cultured and acute slices (Figure 8) overlap and have a similar broad skewed shape. The mean GFP fluorescence intensity for the cultured slices is $0.352 \pm 0.007(\mathrm{n}=566)$ and for the acute slices (data from Suzuki and Bekkers 2010), the mean is $0.352 \pm 0.002(\mathrm{n}=14180)$. These values are equal considering uncertainty and that different people took the recordings, which suggests that the GABAergic interneurons are not changed in the culture.

This similarity is further demonstrated in the soma size histograms, which also overlap (Figure 9). The overlap is not perfect, which suggests some change, though this is possibly due to the acute and cultured slice data being collected by different people. The means, however, are almost identical, for the cultured slices the mean is $134.0 \pm 3.7 \mu \mathrm{m} 2(\mathrm{n}=566)$ and for the acute slice is $133.2 \pm 0.4 \mu \mathrm{m} 2$ $(\mathrm{n}=14180)$. Therefore, it is likely that the size of the GABAergic interneurons is not affected by the culture and that no particular subtype has been lost. 


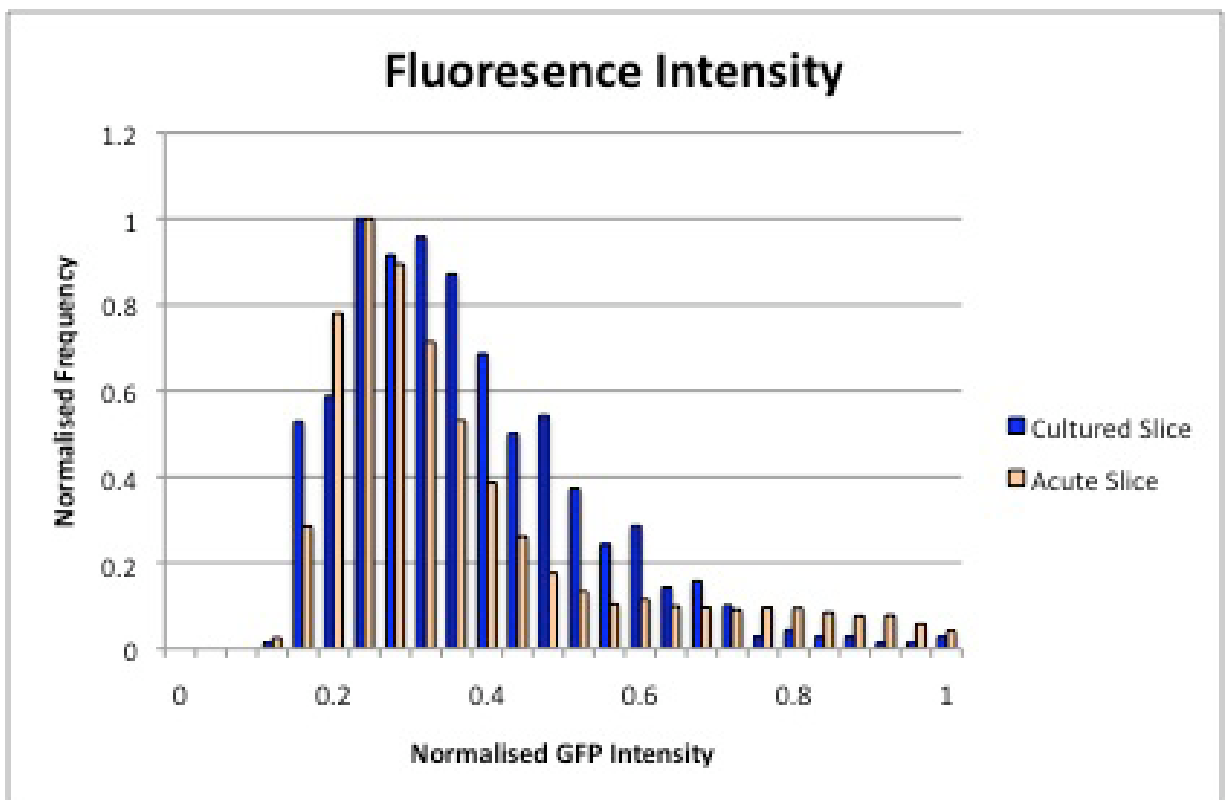

Figure 8: Normalised frequency histograms of the normalised GFP intensity in the cultured and acute slices.

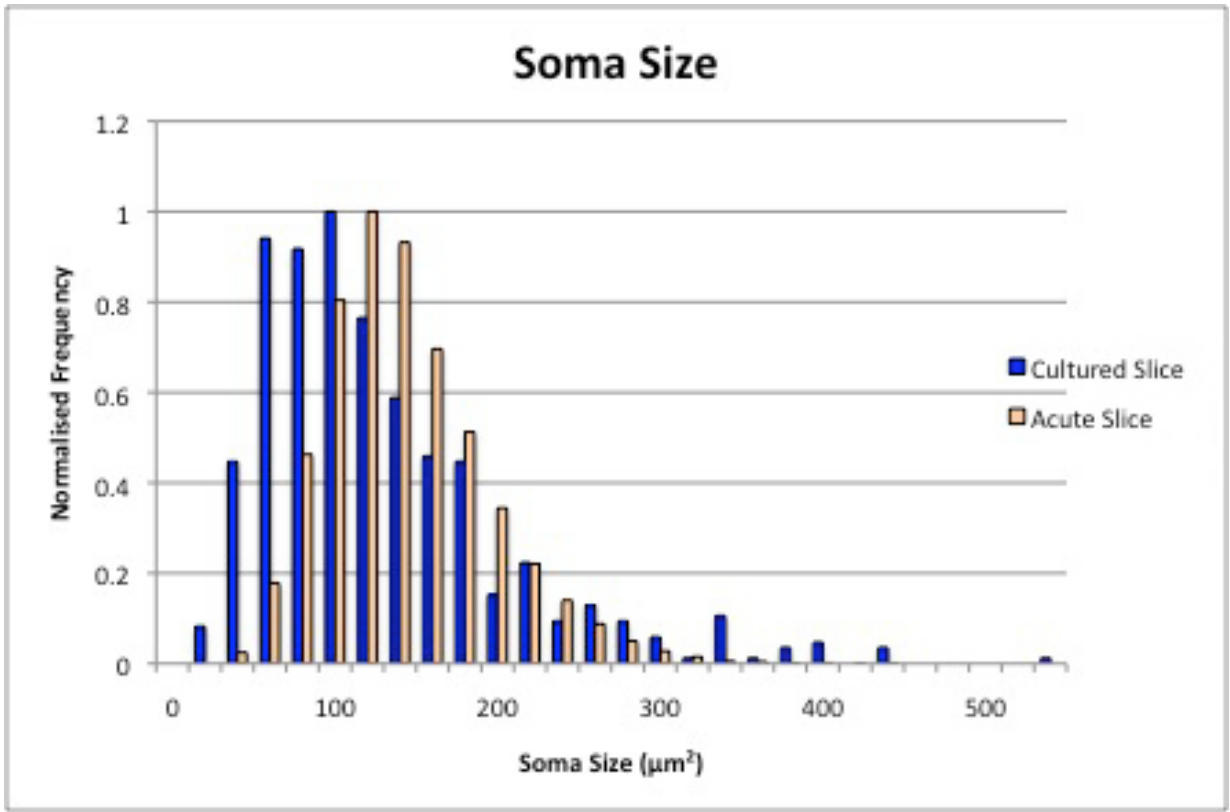

Figure 9: Normalised frequency histograms of soma size in the acute and cultured slices 


\section{Discussion}

From this study it appears feasible to culture parasagittal slices containing the OB, LOT and the PC but that there may be some differences. We have been the first to apply this method to parasagittal slices for use in studying the olfactory system. To ensure as many potential differences between the slices were identified, a range of different methods of analysis were applied. Since these slices will be used in connectivity studies, the main focus was on the morphological, intrinsic and synaptic properties of individual neurons in the PC, as well as survival of GABAergic interneurons (believed to play an important role in the processing of information in the olfactory system (Suzuki and Bekkers, 2010).)

The culture method used in this study was based on that reviewed by Gähwiler et al. (1997) and first applied to the hippocampus by Stoppini et al. (1991). Studies, in different areas of the brain, demonstrated that cultured slices are similar enough to acute slices. Our study found differences in the slices, suggesting this method might not be of a suitable standard for studying the olfactory system. We recommend groups studying other cortical areas to also investigate these differences to ensure that their future findings are not merely an artefact of the culture method.

If the results of further studies reflect those found here, then we could conclude that these slices are suitable for use in some research as long as the differences are considered.

\section{The morphology of the putative pyramidal neurons requires further analysis.}

The differences in the morphology of the stained neurons in the acute and cultured slices suggests that changes may be occurring in culture, however, with the small sample size this may just be a result of the diversity of structure between pyramidal neurons (Kandel 2000). More cells need to be traced and their differences quantified (for example by calculating the average number of spines on the dendrites) before any changes can be considered due to the culture. One hypothesis is that one of these neurons was an interneuron, which explains the differences in the electrophysiological properties.

\section{After the slices have been in culture the intrinsic properties of the pyramidal neurons change.}

In this study we found differences between the intrinsic properties of the neuron in the acute slice and those in the cultured slices. This may be due to the culture method, differences in the quality of the patch recording or just interneuronal variability. 
The increase in resting membrane potential in the cultured slice neurons means that they would be more depolarised and thus more excitable. This is consistent with reduced synaptic latency. The increase in input resistance after culture suggests that there has been a decrease in the number of ion channels. This is consistent with the finding that in culture, neurons tend to simplify and strengthen their connections (Gähwiler 1997), which would result in a reduction in ion channels. This could also be the result of the difference in age of the slices during analysis (25 days for acute, 14 days for cultured). As neurons age, they produce more connections and more channels (Kandel, 2000) which lowers the resistance.

The difference in spike width between the neurons in the acute and cultured slices is interesting, as we expect the spike width to be the same for neurons exposed to similar conditions (Stuart, 1997). This supports our earlier hypothesis that we were recording from different types of neurons. However, it is also possible that this was due to a change in conductance of the $\mathrm{K}+$ ion channels. A smaller influx of $\mathrm{K}+$ would slow hyperpolarisation, thus increasing the width of the spike, as seen in the cultured slices (Kandel 2000). This could be explained by an increase in voltage threshold, which would result in it being more difficult for the action potential to fire. A change in the conformation of the resting channels would affect the membrane time constant. Since the membrane time constant and the spike width increased, it suggests that a change in the conformation of the channels has occurred during culture.

If these changes in the intrinsic properties are confirmed by analysing more neurons then the cultured slices are not suitable for electrophysiology studies unless the differences can be quantified and applied during analysis.

The long-distance synaptic connections appear changed in the culture, whereas the short-distance synaptic connections appear similar.

By studying the changes in EPSP patterns after the neurons had been in culture, we found that their long distance (LOT to Layer II) connections had dramatically altered but that the short distance (within Layer II) connections had not.

The synaptic delay is proportional to the number of synapses that the signal has to pass through before reaching the electrode (Kandel, 2000). Since this appeared greater in the neurons from the cultured slices, it is possible that the neurons have decreased the number of long and increased the number of short distance connections. This explains why facilitation of the EPSPs is not observed in the LOT stimulated cultured slice but is observed in the LOT stimulated acute slice. This is also consistent with Gähwiler et al. 2005 review, which hypothesised that intercellular connections rearrange in culture. 


\section{GABAergic interneurons survived the culture process}

The fluorescence analysis suggested that none of the GABAergic interneuron subtypes present in the acute slices were absent from the cultured slices This distictinon was used by Bekkers and Suzuki (2010) to classify the subtypes. The slight difference in the shapes of the histograms could be attributed to different experimenters recording the results. Since the means are equal within uncertainties, it is reasonable to assume that there is little change in the relative numbers of each type of GABAergic interneuron. This suggests that there is no specific loss of subtypes of these cells, in culture. This result, combined with our finding that there are minimal changes in short distance connectivity, implies that the processing of information within the PC could be studied using these slices. To ensure all types of neurons survive, further histological studies would need to be completed. Also, it would be useful to study the total number of neurons to ensure there is not an overall loss.

\section{Conclusion}

The use of cultured slices in understanding the connectivity and molecular biology of the olfactory system is feasible but there are some limitations in this technique. Future studies with larger sample sizes are needed to quantify the differences outlined in this paper. The culturing of slices is a useful technique that could be applied to other areas of the brain but it is important to complete a thorough comparative study on the slices before use.

\section{Acknowledgments}

For his ongoing support and assistance in the completion of this project and for inspiring me with his passion for Neuroscience, I would like to sincerely thank John Bekkers. I would also like to acknowledge the help of Norimitsu Suzuki in the laboratory and Anna Cowan in the editing process.

\section{References}

Brann J., Saideman S., et al. (2007). "Strategies for odor coding in the piriform cortex." Journal of Neuroscience 27: 1237-1238.

Buck L., and Axel R. (1991). "A novel multigene family may encode odorant receptors: A molecular basis for odor recognition" Cell, vol. 65, 175-187 
Dennis C. (2004). “The Sweet Smell of Success." Nature 428: 362-364.

Firestein S. (2005). "A Nobel Nose: The 2004 Nobel Prize in Physiology and Medicine." Neuron 45: 333-338.

Gahwiler B., Capogna M., et al. (1997). “Organotypic slice cultures: a technique has come of age." Trends in Neuroscience 20(10): 471-477.

Horikawa A. and Armstrong W. (1988) "A versatile means of intracellular labeling: injection of biocytin and its detection with avidin conjugates." Journal of Neuroscience Methods 25: 1-11

Stuart G., Schiller J., et al. (1997). "Action potential initiation and propagation in rat neocortical pyramidal neurons." Journal of Physiology 505(3): 617-632.

Kandel E., Schwartz J., et al. (2000). Principles of Neural Science, McGraw Hill.

Poo C. and Issacson J. (2009). "Odor Representations in Olfactory Cortex: "sparse" coding, global inhibition, and oscillations." Neuron 62: 850-861.

Shepherd G. (2006). "Smell images and the flavour system in the human brain." Nature 444: 316-321.

Stettler D. and Axel R. (2009). "Representations of odor in the piriform cortex." Neuron 63: 854-864.

Stoppini L. et al. (1991). "A simple method for organotypic cultures of nervous tissue." Journal of neuroscience methods 37: 173-182.

Suzuki N. and Bekkers J. (2010). "Distinctive classes of GABAergic interneurons provide layer-specific phasic inhibition in the anterior piriform cortex." Cerebral Cortex (in press). Published online May 10, 2010. 10.1093/cercor/ bhq046

Suzuki N. and Bekkers J. (2010). "Inhibitory neurons in the anterior piriform cortex of the mouse: classifcation using molecular markers." The Journal of Comparative Neurology 518: 1670-1687.

Suzuki N. and Bekkers J. (2006). "Neural coding by two classes of principal neurons in the mouse piriform cortex." Journal of Neuroscience 26(46): 11938-11947.

Wilson D. and Stevenson R. (2003). "The fundamental role of memory in olfactory perception." Trends in Neuroscience 26(5): 243-248. 